\title{
Corpus
}

$21 \mid 2020$

Dispositifs numériques et dévoilement de soi

\section{Réagir au dévoilement de soi dans un forum de discussions pour les personnes vivant avec un cancer : une approche interactionnelle}

Responding to self-disclosure in an online discussion forum for people living with cancer: an interactional approach

Olivier Turbide, Maria Cherba et Vincent Denault

\section{(2) OpenEdition}

Journals

\section{Édition électronique}

URL : http://journals.openedition.org/corpus/4987

DOI : $10.4000 /$ corpus.4987

ISSN : $1765-3126$

\section{Éditeur}

Bases; corpus et langage - UMR 6039

\section{Référence électronique}

Olivier Turbide, Maria Cherba et Vincent Denault, « Réagir au dévoilement de soi dans un forum de discussions pour les personnes vivant avec un cancer : une approche interactionnelle », Corpus [En ligne], 21 | 2020, mis en ligne le 09 mars 2020, consulté le 24 janvier 2021. URL : http://

journals.openedition.org/corpus/4987 ; DOI : https://doi.org/10.4000/corpus.4987

Ce document a été généré automatiquement le 24 janvier 2021.

(c) Tous droits réservés 


\section{Réagir au dévoilement de soi dans un forum de discussions pour les personnes vivant avec un cancer : une approche interactionnelle}

Responding to self-disclosure in an online discussion forum for people living with cancer: an interactional approach

Olivier Turbide, Maria Cherba et Vincent Denault

\section{Introduction}

1 Depuis les années 2000, plusieurs études portant sur les plateformes de soutien social en ligne destinées à des personnes vivant avec une maladie chronique ont montré que les soutiens demandés ou offerts sur ces plateformes renvoient principalement au soutien informationnel et au soutien émotionnel (Eichhorn 2008 ; Fukkink 2011; Mo et Coulson 2008). Centré sur le partage d'informations, de conseils ou de recommandations, souvent afin de prendre des décisions ou de résoudre des problèmes (Finn 1999), le soutien informationnel définit un cadre interactionnel relativement formel, associé à l'interaction de services bénéficiaire/professionnel. Il s'articule autour de l'expression d'un besoin de soutien (question, souhait, demande), et de réponse(s) à ce besoin, que ce soit par l'évaluation la situation vécue par le demandeur ou par la formulation d'un conseil, par le partage d'une information, ou par les deux à la fois (Gauducheau et Marcoccia 2011). Pour le soutien émotionnel qui renvoie à un apport de réconfort, de sympathie, d'encouragement ou d'affection (Barrera et al. 2002), la demande de soutien demeure souvent implicite, contrairement au soutien informationnel. Il exige des interlocuteurs la réalisation d'un travail interprétatif important afin d'inférer avec justesse le besoin sous-jacent au récit d'émotions, d'expériences, de sentiments, révélant la complexité de son accomplissement. 
2 Or, à notre connaissance, peu de travaux se sont intéressés dans une perspective d'analyse du discours et des interactions (Kerbrat-Orecchioni 2009 ; Clayman et Teas Gill 2012 ; Paulus, Warren et Lester 2016) à l'étude fine des réactions à ces interventions marquées par le dévoilement de soi et aux défis qu'elles posent sur le plan de l'interaction. Considérant la part significative de celles-ci sur les plateformes de soutien social en ligne (Barak et Gluck-Ofri 2007), il y a lieu de s'interroger : 1) sur la mise en discours du dévoilement de soi ; 2) sur les réponses que les membres de la communauté apportent à ces contributions; et 3) le cas échéant, sur l'évaluation satisfaisante (ou non) du soutien. Dans cette recherche, nous proposons d'explorer ces trois questions, à partir d'outils développés en analyse des interactions en ligne (voir Meredith et Potter 2014).

\section{Le dévoilement de soi sur les forums de soutien social en ligne}

3 Comme le rappelle Traverso (1996), le dévoilement de soi constitue une activité interactionnelle qui, d'une part, est centrée sur la personne qui se dévoile, qui rend compte d'un besoin de s'exprimer, de se comprendre (soi-même), de partager certaines situations et émotions ${ }^{1}$, et qui, d'autre part, est tournée vers autrui, dans une logique de validation de soi, de reconnaissance et de normalisation de l'expérience vécue. Il s'agit d'une interaction qui fonctionne sur le mode de l'accord, de la co-orientation argumentative et de la réciprocité, invitant l'interlocuteur à partager des expériences similaires à celles vécues par la personne qui se dévoile, à produire des évaluations coorientées de la situation présentée, à demander des précisions et à formuler des encouragements (Pudlinsky 2005). Plusieurs recherches analysant des plateformes de soutien social en ligne destinées à différentes populations, que ce soit pour des parents (Haugh et Chang 2015), des adolescents dépressifs (Kaufman et Whitehead 2018) ou des femmes atteintes de problèmes du cancer du sein (Pistrang, Solomons et Barker, 1999), ont montré que les réactions au dévoilement de soi marquées par l'empathie, la compréhension et l'écoute sont celles préférées. D'ailleurs, il ressort que les réactions davantage intrusives et prescriptives (p.ex. les conseils), constituant une offense territoriale selon les termes de Brown et Levinson (1987), ne seront acceptées que si elles sont introduites par des marques d'empathie et de solidarité (Morrow 2006) provenant d'un membre se montrant similaire à la personne qui se dévoile (Pudlinski 2002).

4 Même si le bien-être psychologique qu'elles procurent semble moins évident ${ }^{2}$, les réactions prescriptives aux partages d'émotions négatives sont prépondérantes sur plusieurs plateformes étudiées (Morrow 2006; Smithson et al. 2011 ; Vayreda et Antaki 2009). Pour Smithson et ses collaborateurs (2011) qui ont analysé les réactions aux récits en ligne de personnes révélant s'être mutilées, cette préférence pour le conseil s'explique en partie par les contraintes et attentes associées aux espaces de soutien social en ligne. En ce sens, dans le cadre d'une étude des interactions sur un forum de soutien aux personnes atteintes d'un trouble bipolaire, Vayreda et Antaki (2009) posent l'hypothèse que ces réactions prescriptives non sollicitées renvoient à une culture de l'intervention sur ces plateformes où le soutien social est envisagé comme une aide à la résolution de problèmes ${ }^{3}$. Le rôle joué sur ces plateformes par les membres les plus actifs - représentant en moyenne $10 \%$ des membres mais produisant de $50 \%$ à $70 \%$ des 
messages (Akrich et Méadel 2009) - semble être un autre facteur explicatif de cette prédominance du conseil. Invités à agir à la fois comme agent de socialisation, recommandant aux nouveaux membres des comportements à adopter sur le forum (Stommel et Koole 2010) et comme " gardien » des valeurs et des normes en partage au sein de la communauté en ligne (Vayreda et Antaki 2009), ces «leaders" sont ainsi responsables d'une part significative des conseils que l'on retrouve sur ces plateformes. Plus que la valorisation et la reconnaissance qu'ils obtiennent du fait de conseiller autrui, cette activité prescriptive participe aussi à la construction de leur légitimité et de leur autorité sur le forum (Pertersen et Smithson 2010).

En filigrane de ces résultats apparaît une contradiction entre les réponses attendues au dévoilement de soi, soit l'empathie, la compréhension et l'écoute, et la prédominance d'un type d'actes (prescriptifs), qui introduit une asymétrie entre la personne qui se dévoile et celle qui offre le soutien. Bien que certains facteurs aient été explorés pour expliquer cette contradiction (contraintes sociotechniques des plateformes, conception normative du soutien social, construction de l'identité du groupe), nous émettons l'hypothèse que ce décalage entre les réactions attendues et produites prendrait source également dans l'attribution d'un sens différent à l'activité de soutien en cours. Chacun, demandeur et offreur de soutien, activerait un cadre interactionnel (Goffman 1974) différent pour appréhender l'échange; une différence de cadrage laissant des traces dans les échanges en ligne que l'analyse des interactions pourrait faire émerger.

\section{Méthode}

Dans une perspective socio-interactionniste du discours qui intègre les acquis de l'analyse conversationnelle, de l'ethnométhodologie et de l'ethnographie de la parole, l'analyse des interactions proposée, par la prise en compte de la construction séquentielle des échanges et de sa gestion par les interlocuteurs, permet d'appréhender efficacement la façon dont les épisodes de dévoilement de soi lors de l'ouverture d'un fil de discussion sont interprétés et répondus par les autres membres du forum. Envisageant chacune des interventions d'un fil comme interreliée et interdépendante, cette analyse micro des interactions repose sur le postulat que la construction locale des échanges numériques est socialement organisée et s'arrime à un type d'activité socialement défini et contraint à un niveau macro (Sacks [1964-1972] 1992). Sur des plateformes de soutien social en ligne comme celle étudiée ici, l'expérience commune et partagée des membres rend compte d'un cadre interactionnel qui - même s'il est adapté in situ- définit des comportements attendus et désirés, orientant l'interprétation des contributions en termes de demande et d'offre de soutien.

7 Deux principes méthodologiques développés par Schegloff et Sacks (1973) en analyse conversationnelle complètent ce postulat d'une articulation entre le niveau micro - de la gestion interactionnelle des échanges - et le niveau macro - de la négociation des activités sociales réalisées. Le premier de ces deux principes est celui de la dépendance séquentielle voulant que tout tour de parole, tout "message », en raison du type d'acte qu'il réalise, prescrive un type d'acte attendu et contextuellement pertinent et offre un espace interactionnel aux interlocuteurs pour le réaliser. Ainsi, la formulation d'une question génère une attente forte que le tour suivant constitue une réponse à cette question, plaçant les destinataires de la question devant cette obligation à la fois morale et sociale de réagir en donnant l'information demandée. De même, l'offre de 
soutien social constitue le comportement attendu et socialement désiré à toute forme de demande de soutien. Tout écart à cette contrainte de dépendance des tours de parole est susceptible de nuire à l'accomplissement de l'échange. Cela ne signifie pas qu'une demande d'information qui ne reçoit pas une réponse informative, par exemple, soit interactionnellement non pertinente. Cela signifie que l'interlocuteur n'a pas interprété l'intervention précédente comme une demande d'information, mais comme une invitation à partager une expérience, par exemple. Toutefois, le locuteur qui a formulé clairement sa question risque de sortir frustrer de son échange, ses attentes à l'égard d'une réponse à sa question n'étant sans doute pas satisfaites. Et, c'est là un (autre) principe central de l'analyse des interactions, le deuxième qui informera notre analyse, selon lequel l'intervention réactive révèle l'interprétation et la valeur que l'interlocuteur accorde à l'intervention précédente.

8 Appliquée aux trois questions de cette recherche, notre analyse de la gestion des interactions et des actes de discours qui les composent permettra d'évaluer comment les membres interprètent et négocient les épisodes de dévoilement de soi, comme une activité spécifique de dévoilement de soi qui appelle une réaction d'écoute, de prise en compte l'autre, d'empathie ou comme une activité de soutien de type «conseil » qui appelle la prescription de comportements ou d'attitudes. Complétant cette analyse des interactions, une analyse de contenu a été réalisée afin de caractériser les interventions initiales en fonction de la nature de l'information dévoilée (factuelle ou émotionnelle) et de l'orientation de celle-ci pour la personne qui se dévoile (positive - bonnes nouvelles; négatives - mauvaises nouvelles; mitigée - ni bonnes, ni mauvaises, ou neutre), et les interventions réactives selon que les actes réalisés lors de ses interventions soient de nature prescriptive, empathique ou autres.

\section{Corpus}

Notre corpus est tiré d'un forum de discussion pour soutenir les personnes vivant avec le cancer et leurs proches. Développée par la Société canadienne du cancer, la plateforme d'échange entre pairs étudiée [ParlonsCancer.ca] comptait 2214 participants inscrits en mars 2017, dont 80 qui participent aux échanges analysés. Comme dans d'autres forums étudiés (Akrich et Méadel 2009), la plupart des messages sont écrits par un noyau très restreint de participants plus actifs. En mars 2017, par exemple, $54 \%$ des messages du forum ont été écrits par les quatre participants les plus actifs. Ainsi, la majorité des participants est constituée de lecteurs (lurkers).

10 Au total, 44 fils de discussion s'amorçant par une intervention de dévoilement de soi ont été sélectionnés à partir d'un corpus initial constitué 161 fils créés par les participants du forum sur quatre mois, sélectionnés au hasard à tous les 4 mois entre 2017 et 2018. En plus d'appartenir au discours de dévoilement de soi selon la caractérisation de Traverso (1996) citée précédemment, les messages initiaux des fils sélectionnés devaient porter spécifiquement sur le partage d'expériences liées au cancer et ne devaient pas inclure ni d'acte de question (c'est-à-dire des énoncés interrogatifs syntaxiquement marqués par des mots-questions (wh-questions) ou des énoncés assertifs à valeur interrogative), ni d'actes de demande d'information ou de soutien et ni d'énoncés exprimant un souhait ou un besoin.

11 Ces 44 fils de discussion comprennent 344 messages au total et s'inscrivent en grande majorité dans une structure d'échange. Seuls quatre fils introduits par un message de 
dévoilement de soi n'obtiennent aucune réponse. La moitié (22 sur 44) des fils de notre corpus sont initiés par des participants qui interviennent pour la première fois sur le forum alors que ce sont surtout des participants plus anciens et plus actifs qui répondent.

\section{Considérations éthiques}

12 Même si le forum étudié est public - tout internaute peut avoir accès aux contributions publiées sur le forum et le nombre de participants est suffisamment important (Eysenbach et Till 2001)-, afin de rendre explicite notre présence et informer les participants de la cueillette de données que nous projetions de faire, nous avons publié un message sur la plateforme à propos des objectifs du projet de recherche et invité les participants à nous demander, s'ils le souhaitaient, de retirer leurs contributions du corpus d'analyse. Au terme de cette démarche, nous avons retiré du corpus les messages produits par un participant.

13 Conscients des risques associés à la publication d'extraits des fils de discussion, à l'instar de Vayreda et Antaki (2009), nous avons modifié les pseudonymes des participants du forum. De plus, nous avons supprimé ou modifié les informations permettant d'identifier un participant (p.ex., nom d'un lieu, d'une institution, d'une personne). Par ailleurs, parce que l'objet de recherche est les processus fins d'interaction entre les participants de ce forum, il nous est apparu primordial de conserver les messages sans altérer le sens du discours, tout en les modifiant légèrement pour qu'ils ne puissent pas être retracés à l'aide des moteurs de recherche (correction orthographique, correction des mots en phonétique).

\section{Analyse}

14 À l'encontre de conclusions d'études précédentes (Morrow 2006; Smithson et al. 2011; Vayreda et Antaki 2009) où le conseil apparaît comme une réaction privilégiée au dévoilement de soi, notamment quant à l'exposition de problèmes, les résultats de notre étude sont davantage nuancés. De fait, les actes d'empathie (relancer, encourager, montrer une écoute, une compréhension, partager une expérience similaire) sont dominants, représentant $53,1 \%(434 / 817)$ de l'ensemble des actes produits lors des interventions réactives. Cela étant dit, les actes de prescription de comportements ou d'attitudes, prenant la forme surtout de conseils et de partages d'expérience à visée prescriptive ${ }^{4}$, demeurent importants et représentent un peu moins $\mathrm{du}$ tiers des actes, avec 32,4\% (265/817). Le reste des actes, marques d'humour, information, évaluation du comportement sans prescription, ni marques d'empathie, occupe pour leur part $14,4 \%$ (118/817). Une analyse plus fine de ces résultats quantitatifs montre que les actes prescriptifs ne sont pas concentrés dans certains fils, mais apparaissent ici et là, dans $82,5 \%(33 / 40)$ des fils avec structure d'échange, aux côtés d'actes empathiques.

\section{Dévoilement de soi et prescription de comportements ou d'attitudes}

15 La nature de l'information dévoilée et son orientation influencent le type de soutien offert comme le montrent les résultats présentés au Tableau 1. De fait, le dévoilement 
d'émotions négatives commande davantage de réactions de prescription que le dévoilement d'émotions positives ou celui d'informations factuelles, positives ou négatives. Lorsqu'un membre partage des émotions négatives, il reçoit en moyenne 1,6 acte de prescription de comportements par message alors que ce ratio est seulement 0,9 acte de prescription par message lorsqu'il partage des informations négatives. Peu de conseils, recommandations ou autres prescriptions sont prodigués lorsqu'une information positive est dévoilée, qu'elle soit de nature émotionnelle $(0,3$ acte de prescription/message) ou informationnelle (0,2 acte de prescription/message). Inversement, ce sont les informations négatives, émotionnelles et informationnelles, ainsi que les informations émotionnelles positives qui génèrent davantage de comportements empathiques.

Tableau 1. Fréquence d'actes par intervention réactive en fonction de la nature et de l'orientation de l'intervention initiale

\begin{tabular}{|l|c|c|c|c|c|c|}
\hline $\begin{array}{r}\text { Intervention } \\
\text { initiale }\end{array}$ & \multicolumn{3}{|c|}{ Information factuelle } & \multicolumn{3}{c|}{ Information émotionnelle } \\
\cline { 2 - 7 } $\begin{array}{l}\text { Intervention } \\
\text { réactive }\end{array}$ & Positive & Négative & $\begin{array}{c}\text { Mitigée/ } \\
\text { Neutre }\end{array}$ & Positive & Négative & $\begin{array}{c}\text { Mitigée/ } \\
\text { Neutre }\end{array}$ \\
\hline Actes de prescription & 0,2 & 0,9 & 0,3 & 0,3 & $\mathbf{1 , 6}$ & 1,0 \\
\hline Actes d'empathie & 1,3 & $\mathbf{2 , 2}$ & 1,6 & $\mathbf{2 , 7}$ & $\mathbf{2 , 1}$ & 1,8 \\
\hline Autres types d'actes & 1,2 & 0,3 & 0,1 & 1,7 & 0,5 & 0,6 \\
\hline
\end{tabular}

Cette interprétation voulant que le partage d'émotions négatives appelle une réaction de prescription est particulièrement saillante dans les fils initiés par le dévoilement d'une situation présentée comme émotionnellement insurmontable, associée à du découragement ou de la tristesse et à de la colère ou de la révolte. L'analyse interactionnelle de l'enchainement à ce type d'interventions initiales montre que les membres y réagissant interpréteront moins ce dévoilement comme une invitation à l'écoute et à la compréhension que comme l'expression d'un besoin de soutien pour mieux gérer les émotions exprimées. Dans ce contexte, comme à l'extrait 1 , les émotions extrêmes ressenties par la personne qui se dévoile seront alors ratifiées, puis recadrées afin de l'orienter vers l'adoption d'une attitude positive face à la maladie et davantage conforme au crédo du forum (« Un jour à la fois ») $)^{5}$. En outre, l'absence de prise en charge énonciative des énoncés par L2 leur attribue une valeur d'évidence qui accentue l'asymétrie entre quelqu'un qui sait, qui a l'expérience, et quelqu'un qui ne sait pas, propre à l'interaction de services bénéficiaire/professionnel.

\begin{tabular}{|l|l|l|}
\hline (1) & Fil 47 & \\
\hline L1.47.1 & $\begin{array}{l}\text { Je suis d'une tristesse infinie ! On m'a donné le diagnostic de tumeur cancéreuse à la } \\
\text { vessie ! Je dois être opérée le 2 février. S'ensuivra sûrement chimio ! Ma vie ne sera } \\
\text { jamais plus la même! }\end{array}$ \\
\hline L2.47.2 & $\begin{array}{l}\text { L1, c'est vrai que la vie ne sera plus pareille, vous la verrez sur un nouvel angle, mais } \\
\text { elle sera toujours vôtre. En étant réaliste, essayez de ne pas trop extrapoler sur la suite } \\
\text { des choses. Un jour à la fois suffit sa peine car l'anxiété, même si normale, ne nous } \\
\text { mène à rien. }\end{array}$ \\
\hline
\end{tabular}

La mise en scène discursive de la situation dévoilée semble aussi affecter le type de soutien proposé. En ce sens, il apparaît que le récit d'émotions s'articulant autour de 
l'identification d'un problème, entendu comme une difficulté à résoudre, est susceptible d'être interprété comme une demande de conseil.

\begin{tabular}{|l|l|l|}
\hline (2) & Fil 916 \\
\hline L1.91.1 & $\begin{array}{l}\text { Depuis 2015 que ma femme est suivie pour un cancer du poumon après une lobectomie } \\
\text { du poumon droit en fin 2016, 2017 des métastases ont apparu au cerveau } \\
\text { inopérable [...] Voilà deux semaines ils ont tenté un dernier traitement de Gamma } \\
\text { scalpel sur la métastase qui n'avait pas réagi au traitement mais on dirait que son état } \\
\text { empire. [...] Je ne sais pas vraiment où me garrocher [lancer], vraiment difficile de } \\
\text { la voir à son réveil sur le fauteuil que je lui aie installé au rez-de-chaussée elle se met } \\
\text { à délirer sur des choses que je n'y comprends rien. Vendredi elle était dans une } \\
\text { phase méchante et aujourd'hui elle était sur le cas des enfants qui ne forcent pas trop } \\
\text { pour venir la voir. Je me sens dans un système de vie familiale complètement } \\
\text { dysfonctionnel. Merci de votre écoute. }\end{array}$ \\
\hline L2.91.2 & $\begin{array}{l}\text { Bonsoir L1 } \\
\text { Avez-vous pensé à réunir vos enfants et leur expliquer ce qui se passe réellement avec } \\
\text { leur mère? Il arrive parfois que l'on ne dit pas tout à nos enfants pour toutes sortes de } \\
\text { raisons, nous attendons avant de leur infliger plus de peine. Alors, s'ils ne savent pas } \\
\text { tout, difficile pour eux de comprendre et de réagir comme ils le devraient. Bonne } \\
\text { chance dans votre démarche. } \\
\text { De tout cœur avec vous et votre conjointe. }\end{array}$ \\
\hline
\end{tabular}

Dans cet extrait (2), le problème est posé par L1 dans la dernière portion de son message (en caractères gras). Il exprime une incertitude sur les gestes à poser en regard des soins à apporter à sa femme malade ( "Je ne sais vraiment pas où me garrocher [me lancer]») et sur la façon de faire sens des réactions de celle-ci («je n’y comprends rien »). Cette problématisation de la situation constitue une caractéristique définitoire de l'interaction de services bénéficiaire/professionnel, laquelle s'amorce bien souvent par l'expression d'un problème suivi de la recherche de complément d'information par le professionnel, qui ensuite évalue la situation et adresse un conseil au bénéficiaire (Laforest 2012). Ici, même si la demande de conseil n'est pas explicitée, elle est sousentendue par l'expression d'un manque de savoir expérientiel, générant en quelque sorte une attente, comprise par les autres membres, afin que cette incertitude soit diminuée. Cela étant dit, notons qu'en dépit de cette problématisation explicite, L2 n'enchaîne pas sur ce qui pose problème aux yeux de L1, soit sa difficulté à composer avec les réactions de sa femme, mais plutôt sur un élément périphérique de son discours, soit le peu d'empressement de leurs enfants à venir les visiter. Ce changement d'orientation thématique est caractéristique des fils étudiés où les pairs qui offrent un soutien sont amenés à sélectionner dans la situation dévoilée, la composante sur lequel ils désirent intervenir.

19 À la différence du fil 91 (extrait 2), dans le fil suivant (3), le problème n'est pas décrit explicitement par la personne qui se dévoile, mais il doit être inféré par celle qui offre le soutien.

(3) Fil 156 


\begin{tabular}{|l|l|l|}
\hline L1.156.1 & $\begin{array}{l}\text { Jour } 4 \\
\text { réveil par douleur presque insoutenable. Lancinante, palpitante. } \\
\text { à la respiration ça fait encore plus mal. [...] } \\
\text { faut que je prenne un rendez-vous urgent pour demain. } \\
\text { organiser mon transport au CLSC [Centre local de services communautaires]. } \\
\text { Attendre que ça soit ouvert. } \\
\text { Me procurer la morphine ou dilodil avec ma prescription. } \\
\text { respiration difficile. }\end{array}$ \\
\hline L2.156.2 & $\begin{array}{l}\text { Bonjour L1 } \\
\text { Vous ne l'avez pas facile... Pourquoi ne pas vous rendre directement au CLSC ce } \\
\text { matin? Question de transport? Pour ce qui est de votre prescription, vous savez que } \\
\text { vous pouvez obtenir une livraison à domicile. Il y a toujours moyen de s'arranger } \\
\text { avec le pharmacien. Cela vaut la peine d'essayer. Bon courage. }\end{array}$ \\
\hline L1.156.3 & \begin{tabular}{l} 
Merci, L2, la morphine fait son effet.[...] \\
\hline
\end{tabular}
\end{tabular}

Ici, L2 prend en charge l'entièreté du processus de soutien. À partir des informations dévoilées de façon télégraphique par L1 ("pren[dre] un rendez-vous urgent», « organiser mon transport », " me procurer de la morphine ou dilodil »), L2 évalue la situation, identifie le problème (des difficultés quant à la gestion de tâches impératives pour la santé de L1) et propose un soutien qui prend la forme de suggestions. À la faveur de cet extrait, il apparaît que le dévoilement de soi, sans problématisation, favorise l'activation d'un cadre interactionnel asymétrique bénéficiaire/professionnel alors que la personne qui se dévoile cède aux autres membres le pouvoir d'évaluer sa situation.

Si, dans l'extrait 3, l'identification du problème est ratifiée par L1 qui remercie son interlocuteur, il demeure que la prise en charge de la problématisation par les pairs est toujours risquée considérant l'information contextuelle fragmentaire dont ils disposent pour évaluer la situation de L1. En ce sens, une telle construction du dévoilement de soi, sans clés interprétatives, ouvre la porte à une possible contestation du problème identifié par les pairs et, par le fait même, de la prescription, comme c'est le cas ici dans le fil 23 (extrait 4) où l'intervention initiale que nous reproduisons en partie est composée uniquement d'un échange entre une mère, atteinte d'un cancer, et sa fille.

\begin{tabular}{|c|c|c|}
\hline \multirow[t]{2}{*}{ (4) } & \multicolumn{2}{|l|}{ Fil 23} \\
\hline & L1.23.1 & $\begin{array}{l}\text { Je vous mets la lettre au complet, trop dure pour moi et Serge, aussi en rouge c'est } \\
\text { moi qui réponds. } \\
\text { J'avais beau te demander au téléphone comment tu allais avec tes antibiotiques.. } \\
\text { quand tu appelais j'avais de la visite, je te rappelais, mais tu me rappelais pas, tu ne } \\
\text { me réponds pas tu me parles d'autres choses. Alors non, je ne suis pas traversée } \\
\text { lundi, comment je peux savoir si tu es guérie? Tu n'as pas de cell, demander je peux- } \\
\text { tu y aller [...] }\end{array}$ \\
\hline & L2.23.2 & $\begin{array}{l}\text { Bonjour L1, } \\
\text { J'ai lu attentivement ce que ta fille a écrit et ta réponse. Je crois qu'autant ta fille que } \\
\text { toi, il y a de l'incompréhension de part et d'autre [Problématisation]. Je te dis cela } \\
\text { parce que j'ai vécu la même situation avec mon mari il y a } 20 \text { ans. [...] Alors L1, je te } \\
\text { conseille de t'asseoir avec ta fille et d'avoir une bonne discussion avec elle } \\
\text { [Prescription de comportements] }\end{array}$ \\
\hline
\end{tabular}




\begin{tabular}{|l|l|l|}
\hline & $\begin{array}{l}\text { [reprise de la recommandation de s'asseoir avec sa fille pour avoir une bonne } \\
\text { discussion, avec certaines variations, dans les huit messages suivants] }\end{array}$ \\
\hline L1.23.11 & $\begin{array}{l}\text { [...] Je sais bien que j'ai l'air de me défendre aux yeux de plusieurs, à vrai dire, je vais } \\
\text { vous dire vraiment ce que je pense, je ne me sens aucunement compris sur le site et } \\
\text { je n'écrirai plus sur ce sujet car j'ai tellement de peine de plus voir mon petit fils. } \\
\text { [Rejet de la problématisation et de la prescription de comportements] }\end{array}$ \\
\hline
\end{tabular}

\section{Accomplissement interactionnel des actes de conseil}

Bien que relativement rare dans l'ensemble du corpus, le dernier extrait illustre bien les malentendus possibles créés par le dévoilement de soi, sans problématisation, ni demande explicite de soutien. Bien que ces séquences prescrivent, sur le mode implicite, une réaction empathique, d'écoute et de compréhension, comme l'évoquent Smithson et ses collaborateurs (2011), il reste que le dévoilement de soi constitue une intervention initiale ouverte et peu contraignante sur le plan interactionnel, permettant aux pairs d'activer, selon l'interprétation qu'ils font de la situation, une activité de conseil, une activité d'empathie, ou les deux à la fois, selon le cas.

En dépit de ces considérations, il reste que prescrire demeure un acte difficile à réaliser si l'on veut soutenir sans blesser, ni paraître inadéquat et ce, en raison son caractère intrusif. Searle ([1969]1972:109) rappelle d'ailleurs que « conseiller ce n'est pas essayer de vous faire faire quelque chose [...]. Conseiller, c'est plutôt dire ce que vous avez à faire", révélant une dimension normative et potentiellement menaçante de la prescription. En ce sens, l'analyse fine de la construction de ces interventions montre que bien souvent les actes d'empathie s'affichent comme des précautions interactionnelles permettant de réduire le potentiel menaçant de l'acte de prescription qui suit. Des encouragements, des relances et/ou des marques d'affection pourront conclure ce développement séquentiel, schématisé ici et illustré par la réaction de L2 à l'extrait 5.

Schéma 1. Développement séquentiel typique d'une prescription empathique

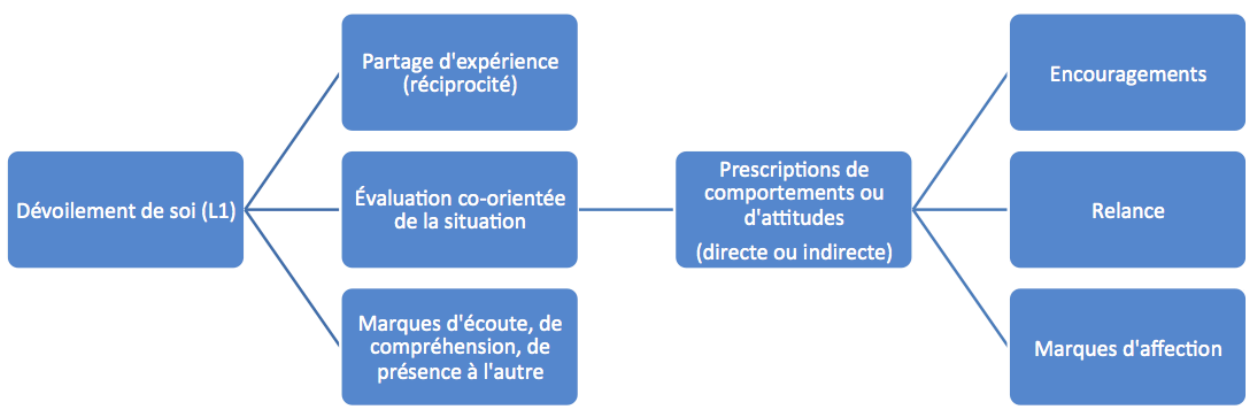

(5) Fil 110

L1.110.1 J'ai subi une mastectomie totale le 2 décembre et je ferai de la chimio et de la radio. J'ai peur des traitements. 


\begin{tabular}{|c|c|}
\hline L2.110.2 & $\begin{array}{l}\text { Bonjour L1, } \\
\text { Je crois qu'avoir peur est tout à fait normal car l'inconnu fait peur... [Évaluation co-orientée de la situation] } \\
\text { Par contre les scénarios que l'on se fait sont parfois pires que la réalité ou complètement différents de ce que l'on a } \\
\text { imaginé. Je viens de passer par la chimio [...] tout s'est bien passé. Je ne vous dis pas que je n'ai pas eu d'effets } \\
\text { secondaires mais je les prenais un à la fois à mesure qu'ils se présentaient [...]. Alors si j'avais amorcé mon } \\
\text { traitement avec la peur de tout ce qui peut arriver, j'aurais été bien malheureuse. } \\
\text { Cela a été une période de ma vie dont je me serais passé mais puisqu'il le fallait, j'ai pris mon petit courage à deux } \\
\text { mains en allant de traitement en traitement [...] en essayant de ne pas me faire trop de scénarios catastrophes. } \\
\text { [Partage d'expériences à visée prescriptive] } \\
\text { Et pour tout vous dire, le fait d'exprimer ici votre peur est très saine, [Évaluation co-orientée] } \\
\text { vous n'êtes pas seule dans ce combat [...] [Encouragement] } \\
\text { "Un jour à la fois, faire son possible, lâcher prise" nous appartient. [Reprise des prescriptions] } \\
\text { Je vous souhaite de rester confiante malgré vos appréhensions [Reprise de la prescription « rester confiante " sous } \\
\text { la forme d'un souhait], } \\
\text { vous venez de passer par une grosse étape avec succès ce n'est pas rien! [Évaluation de la situation / Encouragement] } \\
\text { Bon courage, L2 [Encouragement] }\end{array}$ \\
\hline
\end{tabular}




\begin{tabular}{|c|c|}
\hline \multirow[b]{2}{*}{ L3.110.3 } & cosat \\
\hline & 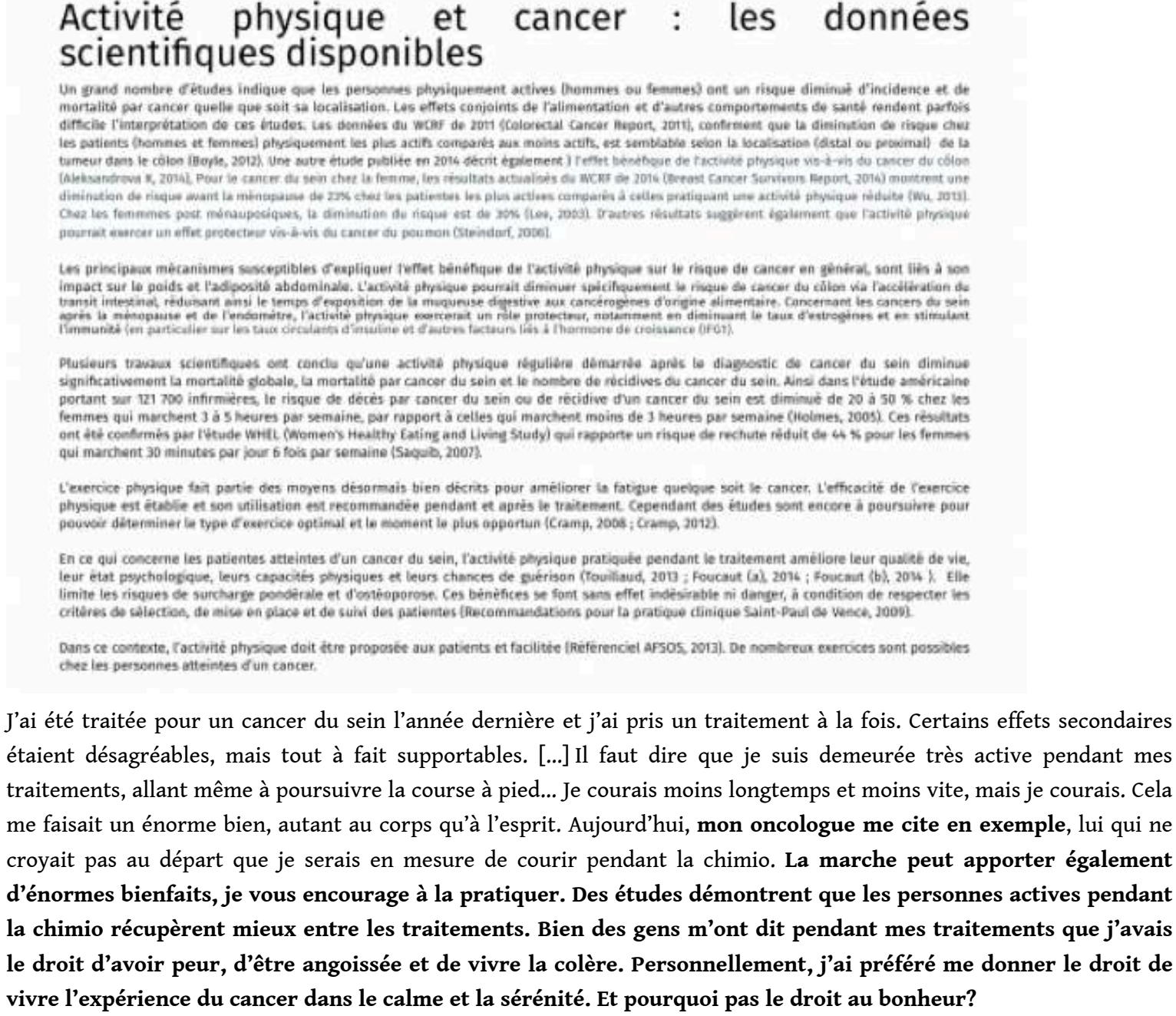 \\
\hline L4.110.4 & $\begin{array}{l}\text { Bonjour L1, } \\
\text { Tout d'abord, nous vous souhaitons la bienvenue sur la plateforme de parlonscancer.ca. Nous comprenons vos } \\
\text { sentiments de craintes et nous espérons que notre famille virtuelle peut vous offrir du soutien. [...] }\end{array}$ \\
\hline L5.110.5 & $\begin{array}{l}\text { Bonjour L1, } \\
\text { Je crois en effet qu'on imagine pire que la réalité, et qu'on se projette trop loin. [...] Avec le cancer, on apprend à vivre le } \\
\text { moment présent et à ne pas imaginer ce que sera demain. [...] }\end{array}$ \\
\hline L6.110.6 & $\begin{array}{l}\text { Bonjour, } \\
\text { Comment se sont passés les traitements? Je pense que le moral fait beaucoup, c'est vrai qu'il y a des hauts et des bas, } \\
\text { mais il faut y croire! Toujours !!! Le fait de bouger et sortir aide beaucoup. Bon courage et donnez-nous des nouvelles }\end{array}$ \\
\hline L7.110.7 & \\
\hline
\end{tabular}

24 La réaction de L2 dans cet extrait (5) rend compte de cette construction séquentielle typique d'un acte de prescription empathique qui s'amorce par l'évaluation co-orientée de la situation de L1 puis, à la faveur du partage de son expérience du cancer, se poursuit par l'expression implicite de conseils (en caractères gras) et se conclut par des marques d'empathie (évaluation positive de son comportement [exprimer sa peur], encouragements). 

convocation d'une autorité scientifique, une capture d'écran d'une synthèse de la recherche sur l'activité physique et le cancer, qui est renforcée par le statut de « modèle à suivre » qu'elle s'octroie (« mon oncologue me cite en exemple »). De plus, la prescription n'est pas indirecte comme pour L2 mais directe : « je vous encourage à la pratiquer [la marche] » et est appuyée par des «études » et par l'expérience de L3. D'autre part, sur le plan thématique, L3 procède également à un recadrage de l'échange, abordant principalement l'activité physique comme stratégie de gestion de la maladie; la peur des traitements qui est la préoccupation de L1 n'étant abordé qu'à la fin du message et récusée en quelque sorte par L3 qui y oppose le «droit au bonheur». À la faveur de ces marques (asymétrie, prescription sans précaution interactionnelle, recadrage thématique), cette intervention qui marque un écart par rapport au développement séquentiel typique d'un conseil empathique témoigne de l'imposition d'une activité prescriptive qui apparait inadaptée à l'intervention de dévoilement de soi de $\mathrm{L}^{7}$.

Cela étant dit et en dépit des stratégies mobilisées par les pairs pour adoucir le caractère intrusif des actes de conseils, le développement séquentiel du fil 110 (extrait 5) illustre comment le cadre interactionnel bénéficiaire/professionnel s’impose à la faveur de la circulation des conseils d'une intervention à l'autre. La reprise de prescriptions morales similaires (vivre le moment présent; rester actif; ne pas anticiper le pire; rester positif), par l'effet de surenchère, introduit une asymétrie entre le nouveau membre et les pairs. Plus encore, leur accumulation à l'intérieur d'un fil conduit à l'attribution d'une valeur de vérité aux prescriptions, pouvant être comprises comme une injonction. La validation des conseils par des membres expérimentés, comme $\mathrm{L} 7$ dans cet extrait, contribue aussi à leur autorité et participe à l'édification des normes de la communauté.

\section{Réactions des L1 aux actes de soutien social}

En regard de la tendance à la prescription de plusieurs interventions réactives qui contrarie d'une certaine façon les attentes d'un soutien empathique associé au dévoilement de soi, nous pourrions penser que les personnes recevant des conseils non sollicités seraient nombreuses à manifester le fait que leurs attentes ne soient pas entièrement comblées. Or, sur les 40 fils contenant une réaction à l'intervention initiale, nous en retrouvons 19 ( $47,5 \%)$ où L1 remercie les pairs pour le soutien ou est en accord avec celui-ci, 19 autres fils (47,5\%) où nous retrouvons aucune rétroaction (ni accord, ni désaccord), et seulement 2 fils ( $5 \%$ ) comportant des marques explicites de refus de la prescription ou de critique. Cette quasi-absence de réactions négatives peut sans doute s'expliquer par le coût interactionnel associé à la production d'une réponse non préférée (soit le reproche au lieu du remerciement), sur le plan à la fois pragmatique, contextuel et émotionnel. Contester un soutien offert, revient à menacer la face des pairs qui offrent le soutien et prendre le risque de se le faire reprocher. C'est également rompre avec la solidarité attendue de toute interaction et plus spécifiquement, de celle attendue par cette communauté en ligne qui définit la solidarité entre pairs comme une norme.

Par ailleurs, si certaines interventions de remerciements ou d'accord de L1 sont conclusives, plusieurs d'entre elles introduisent une boucle de partages d'expériences 
où chaque partage appelle un nouveau partage et ainsi de suite, suivant une logique symétrique de réciprocité qui renforce le cadrage empathique du fil, comme l'extrait 6.

\begin{tabular}{|l|l|l|}
\hline (6) & Fil 44 & \\
\hline L1.44.1 & $\begin{array}{l}\text { Bonjour, } \\
\text { Je suis proche aidante... Ma mère en est à son 2e cancer. [...] L'effet de cette nouvelle a } \\
\text { l'effet d'une bombe sur la personne mais aussi sur la famille. On croit vivre un mauvais } \\
\text { rêve et on pense se réveiller. [...] } \\
\text { Merci de me lire, ce n'est pas tous les jours facile d'accepter qu'on va perdre sa } \\
\text { maman, peu importe notre âge !! }\end{array}$ \\
\hline L2.44.2 & $\begin{array}{l}\text { Bonsoir L1 et bienvenue au sein de notre belle communauté virtuelle. Ce que vit votre } \\
\text { mère est difficile. [...] Il faut vivre plus que jamais un jour à la fois. Continuez de } \\
\text { l'accompagner comme vous le faites. [...] Vous lui apportez beaucoup de bonheur vous } \\
\text { savez. Vous n'êtes pas seule, nous vous accompagnons. } \\
\text { L2 xxx }\end{array}$ \\
\hline L3.44.3 & $\begin{array}{l}\text { Bonjour L1, } \\
\text { je suis tout de cœur avec vous, vous irez lire dans ma présentation, mon père s'est fait } \\
\text { enlever l'estomac en novembre dernier, tumeur cancéreuse. Il doit maintenant faire la } \\
\text { chimio, mais ce n'est pas facile! Nous sommes tellement désemparés face à la maladie } \\
\text { de nos parents, pas facile à digérer. Bon courage, xxx }\end{array}$ \\
\hline L1.44.4 & $\begin{array}{l}\text { Bonjour L3, } \\
\text { Votre situation ressemble à la mienne... les médecins n'ont pas opéré ma mère, elle a } \\
\text { des ganglions à l'extérieur de l'estomac, ce n'était donc pas une solution } \\
\text { envisageable... } \\
\text { On dit un jour à la fois, même si ce n'est pas facile c'est la meilleure chose à } \\
\text { faire... } \\
\text { Merci d'avoir pris le temps de me lire, } \\
\text { Bonne soirée } \\
\text { L1 }\end{array}$ \\
\hline
\end{tabular}

Plus que la réciprocité du partage d'expériences entre L1 et L3, ici, en (6), on remarquera que ces derniers occupent tour à tour le rôle de demandeur de soutien et celui d'offreur de soutien. Ce caractère cyclique et réciproque du soutien n'est pas uniquement le fait de cet échange, mais est récurrent dans les fils analysés et constitue une attente relative au fonctionnement du forum où toute personne qui se dévoile, qui demande du soutien, deviendra éventuellement à son tour une personne ressource offrant du soutien. Cette dynamique, qui n'est pas exclusive à ce forum (voir Barak et Gluck-Ofri 2007), participe d'ailleurs à la socialisation des membres. À ce titre, on notera comment L1, dans sa réaction au soutien offert par L3, intègre le conseil formulé par un membre expérimenté du forum (L2), le fait sien et le reprend et l'adresse à son tour à L3 : «On dit un jour à la fois, même si ce n'est pas facile c'est la meilleure chose à faire... ».

\section{Discussion}

L'analyse détaillée des échanges en ligne sur le forum de la Société canadienne du cancer rappelle la complexité de l'activité de soutien social qui s'exerce sur ces 
plateformes et questionne ce qui fait que l'accomplissement interactionnel de soutien sera jugé satisfaisant (ou pas). En dépit des travaux de Jefferson et Lee (1981) qui établissent une distinction claire entre le dévoilement de problèmes (troubles-talk) et l'interaction de services bénéficiaire/professionnel, nos résultats montrent que les attentes des personnes qui se dévoilent n'apparaissent pas aussi clairement pour les répondants. En effet, si les interventions initiales identifiant clairement un problème en lien avec un écart entre un savoir actuel et un savoir désiré, permettent plus facilement de satisfaire ce besoin par la production de conseils ou de recommandations, dans les autres cas, le soutien souhaité apparaît moins « téléguidé », donnant lieu à de possibles malentendus, comme en (4).

31 Cela étant dit, la représentation prédominante que se font les membres du soutien social, intégrée culturellement, selon laquelle la réponse préférée à un problème ou à une émotion négative demeure la prescription de comportements ou d'attitudes, émerge de notre corpus, comme ce fut le cas pour d'autres terrains de recherche cités précédemment. Dans le cas précis du forum ParlonsCancer.ca, nous pouvons ajouter que l'univers normatif dominant quant à la gestion de la maladie (« une journée à la fois ", " lâcher prise ", " être positif, confiant ») joue également un rôle dans cette posture davantage interventionniste, moins accueillante, pourrions-nous suggérer, pour les émotions négatives "extrêmes", comme la rage, la colère ou le découragement. Cependant, cette tendance au conseil ne se traduit pas par un discours réactif exclusivement centré sur la prescription de façons d'être et de faire, mais s'articule finement, comme nous l'avons montré, à des actes d'empathie, qui permettent d'atténuer la force intrusive du soutien. De même, la prescription, comme acte de discours, se réalise dans une variété de formes, notamment indirectes (par exemple, à travers le partage d'expériences), susceptibles de la rendre plus acceptable et de montrer une proximité expérientielle entre la personne qui se dévoile et celle qui offre le soutien, comme l'a noté Morrow (2006). Cette caractérisation du soutien offert nous a permis de dégager un développement séquentiel qui relèverait de la prescription empathique.

Même en articulant une posture d'écoute, de compréhension et de réciprocité émotionnelle, nous avons noté que ces interventions réactives s'inscrivent pour une large part dans un cadre interactionnel bénéficiaire/professionnel en raison à la fois de l'asymétrie sous-jacente à la production d'une prescription, entre quelqu'un qui sait, qui possède un savoir, et quelqu'un qui ne sait pas, mais également en raison de la circulation de ces prescriptions au sein des fils, où la même prescription est reprise, réaffirmée, confirmée jusqu'à apparaître comme une vérité expérientielle que personne ne remet en doute. Précisons ici que l'absence de mise en doute de ces conseils peut en partie s'expliquer par leur caractère générique. En effet, il s'agit moins de prescriptions contextualisées à la situation de la personne qui se dévoile (son type de cancer, le stade d'avancement de la maladie et des traitements, son âge, etc.), que de prescriptions à large portée à propos de l'attitude positive, résiliente et pragmatique à adopter face à la maladie. Cela permet aux «leaders » du forum d'intervenir sur une base régulière et pouvoir offrir du soutien à un large bassin de personnes atteintes du cancer ou d'aidants.

33 En guise d'ouverture, nous pouvons nous demander, tout d'abord, si ce type de soutien générique est celui qui répond le mieux aux besoins des membres ${ }^{8}$, mais, plus généralement, si le conseil, même empathique, constitue une réponse adéquate et 
susceptible d'offrir un soutien satisfaisant aux personnes qui se dévoilent. $\mathrm{Si}$, à première vue, l'analyse de réactions des personnes qui réagissent au soutien semble permettre de répondre par l'affirmative, la remarque de Smithson et de ses collaborateurs (2011: 498) dans leur analyse d'un forum en ligne de soutien à des personnes révélant s'être mutilées est éclairante: "It is important for forum moderators and researchers to understand that, while advice may ostensibly be asked for, empathy rather than advice is often wanted». Dans la suite de leur article, les auteurs suggèrent que les membres qui participent au forum en tant que demandeur de soutien (même s'ils ne le font pas explicitement), ressortent davantage satisfaits d'une expérience de soutien où ils ont pu se reconnaitre dans le récit des autres et s'identifier à une communauté qui les comprend et qui leur permet de s'exprimer librement et de façon anonyme. Inversement, le soutien social qui prend la forme de prescriptions qui s'affichent comme des vérités expérientielles, est susceptible de créer une asymétrie peu propice au dévoilement réciproque, à l'engagement de (nouveaux) membres et à la participation active au forum.

En outre, il appert que les leaders de ces plateformes, les membres les plus expérimentés et actifs, à l'instar des modérateurs, jouent un rôle central dans la construction de l'identité de ces communautés en ligne et des normes et valeurs qui structurent l'offre de soutien. Il a été permis de constater que ces leaders réagissent souvent les premiers à une intervention initiale et que celle-ci influence les réactions ultérieures, lesquelles renforcent cette première réponse. En ce sens, nous pouvons suggérer que l'adoption de comportements réciproques de la part de ceux-ci, qui tiennent compte de la spécificité de la situation dévoilée, pourrait permettre un accomplissement plus satisfaisant du soutien, susceptible d'améliorer la rétention des membres.

\section{Conclusion}

Par cette analyse des interactions, nous avons voulu contribuer aux travaux actuels qui s'intéressent aux enjeux que pose la réalisation interactionnelle d'une activité de soutien social sur les forums de discussions en ligne. La démarche de recherche visait notamment à mieux outiller les gestionnaires et les modérateurs de ces plateformes afin d'avoir une compréhension fine de la façon dont le soutien est demandé, même de façon implicite à travers le partage d'expériences, de la façon dont ces demandes sont comprises, interprétées et répondues par les pairs et de la façon dont les personnes qui se dévoilent reçoivent (favorablement ou pas) ce soutien. Bien que notre analyse ne permette pas totalement de répondre à la question de la satisfaction, laquelle mériterait d'être prolongées par des entrevues semi-directives des membres à la fois actifs et passifs de cette plateforme, l'exploration des dynamiques d'interactions en ligne pointe vers plusieurs pistes prometteuses relativement à la valorisation d'un soutien plus spécifique et axé sur la réciprocité des expériences et sur la symétrie des échanges, et ce, dans une perspective d'amélioration du bien-être psychosocial des personnes atteintes d'un cancer et de leurs proches. 


\section{BIBLIOGRAPHIE}

Akrich M. et Méadel C. (2009). « Les échanges entre patients sur l'Internet », La Presse médicale 38 : 1484-1490.

Barak A., Gluck-Ofri O. (2007). « Degree and reciprocity of self-disclosure in online forums », CyberPsychology \& Behavior 10(3) : 407-417.

Barrera M., Glasgow R. E., McKay G., Boles S. M. et Feil E. G. (2002). « Do Internet-based support interventions change perceptions of social support ?: An experimental trial of approaches of supporting diabetes self-management », American Journal of Community Psychology 30(5) : 175-194.

Brown P. et Levinson S. (1987). Politeness: Some universals in language usage, Cambridge : Cambridge University Press.

Casili A. et Tubaro P. (2016) Le phénomène « pro-ana ». Troubles alimentaires et réseaux sociaux. Paris : Presses des Mines.

Clayman S. E et Teas Gill V. (2012) «Conversation Analysis », in J. P. Gee et M. Handord (éd.) The Routledge Handbook of Discourse Analysis. New York : Routledge, 120-134.

Denouël J. (2011). « Identité », Communications 88 : 75-82.

Eichhorn K. C. (2008). « Soliciting and providing support over the Internet: An investigation of online eating disorder groups », Journal of Computer-Mediated Communication 14(1) : 67-78.

Eysenbach G. et Till J. E. (2001). « Ethical issues in qualitative research on Internet communities », British Medical Journal 323(7321) : 1103-1105.

Finn J. (1999). « An exploration of helping processes in an online self-help group focusing on issuesof disability ", Health \& Social Work $24: 220-231$.

Fukkink R. (2011). « Peer counseling in an online chat service: A content analysis of social support », Cyberpsychology, Behavior, and Social Networking 14 : 247-251.

Gaudet et al. (2019). "Évaluation du soutien social en ligne sur le forum de ParlonsCancer.ca », Affiche présentée au Congrès 2019 du Programme québécois de cancérologie « Ensemble, en réseau, pour vaincre le cancer ", Montréal (Québec), 22 novembre.

Gauducheau N. et Marcoccia M. (2011). « Le soutien social dans les forums de discussion : réalisations interactionnelles et contrats de communication ", in P. Castel et al. (éd.), Psychologie sociale, communication et langage. Bruxelles : De Boeck, 349-368.

Goffman E. (1974). Frame analysis. Boston : Northeastern University Press.

Haugh M. et Chang W. L. M. (2015). « Troubles talk, (dis)affiliation and the participation order in Taiwanese-Chinese online discussion boards ", in M. Dynel et J. Chovanec (éd.) Participation in public and social media interactions. Amsterdam : Benjamins, 99-133.

Jefferson G. et Lee J. R. (1981). «The rejection of advice: Managing the problematic convergence of a 'troubles-telling' and a 'service encounter' ", Journal of pragmatics 5(5) : 399-422.

Kaufman S. et Whitehead K. A. (2018). «Producing, ratifying, and resisting support in an online support forum », Health 22(3) : 223-239.

Kerbrat-Orecchioni C. (2009). Le discours en interaction. Paris : Armand Colin. 
Laforest M. (2012). « De l'intérêt d'articuler les dimensions discursive et interactionnelle de la conversation. Le cas d'une profession en voie de légitimation », Langue française 175(3) : 91-109.

Meredith J. et Potter J. (2014). « Conversation analysis and electronic interactions:

Methodological, analytic and technical considerations ", in Lim H. L. et Sudweeks F. (éd.)

Innovative methods and technologies for electronic discourse analysis. Hershey : IGI Global, 370-393.

Mo P. K. H. et Coulson N. S. (2008). « Exploring the communication of social support within virtual communities: A content analysis of messages posted to an online HIV/AIDS support group ", CyberPsychology \& Behavior $11: 371-374$.

Morrow P. R. (2006). « Telling about problems and giving advice in an Internet discussion forum: Some discourse features », Discourse Studies 8(4) : 531-548.

Paulus T., Warren A. et Lester J. N. (2016). « Applying conversation analysis methods to online talk: A literature review », Discourse, context \& media $12: 1$-10.

Paveau M. A. (2015). « Extimité », in Dictionnaire de l'analyse du discours numérique. Récupéré de : https://technodiscours.hypotheses.org/category/dictionnaire-dadn.

Pedersen S. et Smithson J. (2010). « Membership and activity in an online parenting community ", in Taiwo R. (éd.) Handbook of research on discourse behavior and digital communication. Hershey : IGI Global, 88-103.

Pistrang N., Solomons W. et Barker C. (1999). « Peer support for women with breast cancer: The role of empathy and self-disclosure ", Journal of community \& applied social psychology 9(3) : 217-229.

Pudlinski C. (2002). « Accepting and rejecting advice as competent peers: Caller dilemmas on a warm line », Discourse Studies 4(4) : 481-500.

Pudlinski C. (2005). « Doing empathy and sympathy: Caring responses to troubles tellings on a peer support line », Discourse studies 7(3) : 267-288.

Rafaeli E. et Gleason M. E. (2009). « Skilled support within intimate relationships », Journal of Family Theory \& Review $1: 20-37$.

Sacks H. ([1964-1972]). Lectures on conversation. Oxford : Blackwell.

Schegloff E. A. et Sacks H. (1973). « Opening up closings », Semiotica 8(4) : 289-327.

Searle J. R. ([1969] 1972). Les actes de langage. Paris : Hermann.

Smithson J., Sharkey S., Hewis E., Jones R., Emmens T., Ford T. et Owens C. (2011). « Problem presentation and responses on an online forum for young people who self-harm », Discourse Studies 13(4) : 487-501.

Stommel E. et Koole T. (2010). « The online support group as a community: A micro-analysis of the interaction with a new member », Discourse Studies $12(3): 357-378$.

Traverso V. (1996) «Dévoilement de soi - Confidence ", in La conversation familière. Analyse pragmatique des interactions. Lyon : Presses de l'Université de Lyon, 194-221.

Vayreda A. et Antaki C. (2009). « Social support and unsolicited advice in a bipolar disorder online forum », Qualitative Health Research 19(7) : 931-942. 


\section{NOTES}

1. Les travaux de Denouël (2011) et Casili et Tubaro (2016) explorent sur d'autres plateformes et dans d'autres contextes institutionnels cette facette du dévoilement de soi qui renvoie à la présentation de soi et dont les pratiques d'«extériorisation de fragments d'intimité », pour reprendre les termes de Paveau (2015 : en ligne), reposent sur un besoin de validation de soi par autrui et sur une recherche de bénéfices personnels et sociaux.

2. Plusieurs travaux montrent que les conseils non-sollicités sont susceptibles d'augmenter le stress, la dépression et le sentiment de solitude de la personne qui les reçoit (voir Rafeli et Gleason 2009).

3. La quasi-absence de conseils non sollicités lors de récits d'expériences positives pointe également en faveur d'une telle conception normative du soutien social (Vayreda et Antaki 2009). 4. «Une personne dans mon entourage a un cancer du sein stade 4 [...]. Elle est toujours là, d'une bonne nature pas compliquée, assez active [...] c'est exactement l'attitude qu'il faut adopter!!! » (Intervention 77.15).

5. Témoignant du caractère identitaire de cette prescription pour les membres $d u$ forum, elle apparait 29 fois dans le corpus de la part de membres expérimentés, mais également de la part de membres intervenant pour la première fois, ayant intégré cette vision du monde à leur discours. D'ailleurs, lorsqu'un nouveau membre mentionne qu'il adopte le «Un jour à la fois», bien souvent les membres y réagiront en renforçant cette disposition, l'évaluant positivement : «L1 - J'essaie de vivre une journée à la fois, mais des fois, au fond de moi, j'ai peur. L2 - [...] Je crois que tu fais déjà la bonne chose VIVRE UN JOUR À LA FOIS» (Fil 14).

6. Dans les extraits, l'usage du caractère gras est de nous.

7. Un indice de ce caractère inadéquat est l'intervention suivante de la modératrice (L4.110.4) qui adopte une posture de compréhension face aux craintes exprimées par L1, rectifiant d'une certaine façon l'intervention précédente. Cela étant dit, L1 ne réagit pas à l'intervention de L3, ni à celles des autres membres d'ailleurs.

8. Selon l'enquête réalisée par Gaudet et ses collaborateurs (2019) auprès d'un échantillon de convenance de 232 personnes (soit 182 personnes atteintes du cancer et 50 aidants), $79 \%$ des personnes atteintes se disent satisfaites du forum de ParlonsCancer.ca et $80 \%$ des aidants.

\section{RÉSUMÉS}

Le dévoilement de soi occupe une part significative des interventions initiales des fils de discussions sur les plateformes numériques de soutien social. Si ce type d'intervention répond au besoin des participants de s'exprimer, de partager leurs émotions, il pose des défis aux interlocuteurs en raison de l'absence de demande explicite de soutien. L'analyse des interactions d'un forum de soutien social en ligne pour personnes atteintes d'un cancer et leurs proches (2017-2018) vise à comprendre comment ce partage d'émotions et d'expériences est interprété et répondu par les pairs, évaluant le cadre interactionnel activé dans de tels contextes. Les résultats montrent que si des actes de prescriptions de comportements et d'attitudes non sollicitées sont présents dans la grande majorité des fils, ils s'articulent finement à des actes d'empathie, qui permettent d'atténuer la force intrusive du soutien. L'analyse révèle aussi comment la formulation du dévoilement de soi influence le soutien offert (empathique/prescriptif). Au final, la présentation des formes d'accomplissement du soutien en réaction au dévoilement de soi 
permet d'envisager des pistes d'action pour favoriser l'engagement et le bien-être psychosocial des membres de ces forums.

Evidence shows the prevalence of self-disclosure statements on online social support platforms. These contributions answer to the members' need to express themselves, to share their emotions. However, it poses challenges for members who respond to such messages because of the absence of an explicit request for support. The main objective of this analysis of messages posted on online social support forum for people living with cancer and their caregivers (2017-2018) is to understand how this sharing of emotions and experiences is interpreted and responded by others members, evaluating the interactive framework activated in such contexts. The results show that acts of prescribing behavior and unsolicited attitudes, present in the vast majority of threads, are often prefaced or followed by acts of empathy, which helps reduce the intrusive force of support. The analysis also reveals how the expression of self-disclosure influences the support offered (empathic/prescriptive). Ultimately, this study seeks to provide strategies of responses for more experienced members to promote a more satisfactory support for those who interact for the first time on the forum and improve their psychosocial wellbeing.

\section{INDEX}

Mots-clés : Soutien social en ligne, personnes atteintes d'un cancer, dévoilement de soi, conseil, empathie

Keywords : online social support, self-disclosure, advice acts, empathy communication, people living with cancer

\section{AUTEURS}

\section{OLIVIER TURBIDE}

Université du Québec à Montréal (UQÀM)

\section{MARIA CHERBA}

Centre de recherche sur la communication et la santé, Université du Québec à Montréal (UQÀM)

\section{VINCENT DENAULT}

Centre de recherche sur la communication et la santé, Université du Québec à Montréal (UQÀM) 